\title{
Curved Dyonic Domain Walls in Four Dimensions
}

\author{
Bobby Eka Gunara \\ Indonesia Center for Theoretical and Mathematical Physics (ICTMP) \\ and \\ Theoretical Physics Laboratory, \\ Theoretical High Energy Physics and Instrumentation Research Group, \\ Faculty of Mathematics and Natural Sciences, Institut Teknologi Bandung \\ Jl. Ganesha No. 10 Bandung \\ bobby@fi.itb.ac.id
}

Received 11-05-2013, Revised 22-08-2013, Accepted 05-09-2013, Published 30-04-2014

\begin{abstract}
In this short paper we show the existence of solitonic solutions of four dimensional ungauged $\mathrm{N}=1$ supergravity coupled to arbitrary vector and chiral multiplets whose Ricci scalar curvature is constant. The Ricci scalar of spacetimes indeed depends on the $\sigma$-model, namely the complex scalars and their first derivative. Then, we give two explicit models, namely static domain walls and static spherical symmetric black holes which are related to our previous works.
\end{abstract}

Keywords: Einstein-Maxwell-Higgs theory, Ricci Scalar, Domain Walls.

\section{ABSTRAK}

Dalam paper ini ditunjukkan eksistensi solusi solitonik dari teori $\mathrm{N}=1$ supergravitasi ungauged berdimensi empat terkopel multiplet vektor dan skalar secara sembarang dimana solusi tersebut mempunyai skalar Ricci konstan. Skalar Ricci tersebut bergantung pada geometri internal medan skalar ( $\sigma$-model) yang kompleks beserta turunan pertamanya. Kemudian, diberikan dua contoh eksplisit, yaitu domain walls statik dan lubang hitam statik bersimetri bola dimana keduanya berkaitan dengan pekerjaan kami sebelumnya..

Kata Kunci: Teori Einstein-Maxwell-Higgs, Ricci Scalar, Domain Walls.

\section{INTRODUCTION}

Topological defects such as domain wall solutions of supergravity have acquired a large interest due to their duality with renormalization group (RG) flows described by a beta function of field theory in the context of AdS/CFT correspondence ${ }^{[1]}$. In particular there has been a lot of study considering these solutions which preserve some fraction of supersymmetry in five dimensional supergravity theory.

Inspired by the development of supergravity theory it is of interest to extend the case to more general theory without supersymmetry. In this short paper, we study solitonic solutions of Einstein-Maxwell-Higgs in four dimensions again inspired by four dimensional $N=1$ supergravity with general gauge-scalar couplings. In particular, we consider a class of solutions called charged domain wall. If the electric and magnetic charges are included in the setup, then the domain walls interpolate two ground states of constant scalar curvature which are not Einstein. These generalize our previous results in neutral domain walls discussed ${ }^{[2-6]}$ in which they connect two Einstein spaces, particularly anti-de Sitter spaces. 
This paper provides our preliminary results on curved dyonic domain walls of four dimensional Einstein-Maxwell-Higgs theory. The "dyonic" means that the solitonic object has both electric and magnetic charges. Moreover, in the theory we should turn on the function called scalar potential of the theory in order to have a domain wall solution.

The structure of the paper can be mentioned as follows. In section 2 we state our general results on charged domain walls. In section 3 we discuss shortly some aspects of four dimensional Einstein-Maxwell-Higgs theory inspired by four dimensional $N=1$ supergravity coupled to vector and chiral multiplets. Then we discuss field equations of motions of the theory in section 4 . Section 5 provides discussion on charged domain walls in the asymptotic regions.

\section{METHOD}

\section{CURVED DOMAIN WALLS}

Now we turn our attention to consider some geometrical aspects of domain walls in (pseudo)Riemannian geometry. In general, the domain wall ansatz metric can be written down as

$$
d s^{2}=\quad \ldots+\quad+\quad,
$$

where $a, b=\quad$ and $a u$ is the warp factor. The components of Ricci tensor have the form

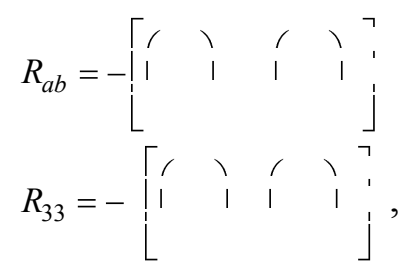

which implies that Ricci scalar is given by

$$
R=-\left[\begin{array}{lllll}
\prime & 1 & \prime & 1 & ? \\
1 & 1 & 1 & 1 & \vdots \\
& & & &
\end{array}\right],
$$

where $a^{\prime} \equiv{ }_{d u}^{J \sim}$. The quantities $\hat{R}_{a b}$ and $\hat{R}$ are the components of Ricci tensor and Ricci scalar in three dimensions, respectively.

Firstly, let us consider a case when the four-manifold is Einstein,

$$
R_{\alpha \beta}=\Lambda_{-,}
$$

where $\alpha \beta=\quad$ and $\Lambda$ is real.

Theorem 1 Suppose we have the condition (5) which follows that the three dimensional hypersurface is also Einstein with constant $\Lambda$. Then, there are only two cases: 
1. For $\Lambda<$, then a $u=\ldots+$ and $A_{0} B_{0}={ }_{4 \Lambda}^{2 \Lambda}$.

2. For $\Lambda>$, then a $u=$ $k^{\prime} \equiv(\quad)$, and $C_{0} D_{0}={ }_{4 \Lambda}^{2 \Lambda}$. where $A_{0}, B_{0}$ are real constants, $k \equiv(\quad)$ ',

where $C_{0}, D_{0}$ are real constants,

Since domain wall interpolates between ground states of different "cosmological" constant in the asymptotic limit, both cases are possible are possible. In the context of field theory the results in Theorem 1 describe an uncharged domain wall ${ }^{[2-6]}$.

Next we turn to a case of constant Ricci scalar

$$
R=\quad .
$$

Theorem 2 Suppose we have the condition (6) such that the three dimensional hypersurface is of a constant scalar curvature with constant $\kappa_{-}$. Then, we also two cases:

1. For $\kappa<$, then $a^{2} u={ }_{\kappa}+\quad+\quad$ where $A_{0}, B_{0}$ are real constants and $k \equiv(\quad)$

2. For $\kappa>$, then $a^{2} u={ }_{\kappa}+\quad+\quad$ where $C_{0}, D_{0}$ are real constants and $k^{\prime} \equiv(\quad)$

Similar as in Theorem 1, the results in Theorem 2 describe a charged domain walls in the asymptotic regions interpolating between the spaces of constant scalar curvature which are not Einstein.

\section{EINSTEIN-MAXWELL-HIGGS THEORY}

In this section we give a short description of Einstein-Maxwell-Higgs theory in four dimensions. This theory is inspired by bosonic parts of $N=1$ supergravity coupled to arbitrary vector and chiral multiplets in four dimensions. For interested reader, the complete $N=1$ supergravity can be found, for example, in ${ }^{[7]}$.

Let us now discuss the ingredients of Einstein-Maxwell-Higgs theory in four dimensions. This theory consists of a gravity coupled to $n_{v}$ vector and $n_{c}$ real scalar fields. Furthermore, the Lagrangian of the theory has the form

$$
\begin{aligned}
L= & -\frac{1}{2} R+g_{i j} \square_{\mu} z^{i} \square z^{j} \\
& +\square \Lambda \Sigma F_{\mu \nu}^{\Lambda} F^{\Sigma \mu \nu}+\square_{\Lambda \Sigma} F_{\mu \nu}^{\Lambda} \tilde{F}^{\Sigma \mu \nu}-V(z)
\end{aligned}
$$


where $i, j=\quad$ and $\Lambda \Sigma=$. The real scalars $z^{i}$ span a Riemannian manifold with metric $g_{i j}$. The quantity $F_{\mu \nu}^{\Lambda}$ is an Abelian field strength of $A_{\mu}^{\Lambda}$, and $\tilde{F}_{\mu \nu}^{\Lambda}$ is a Hodge dual of $F_{\mu \nu}^{\Lambda}$. The functions $\Re_{\ldots}$ and $\mathfrak{I}_{\ldots}$ are real gauge couplings which depend on the scalars $z^{i}$. The real function $V(z)$ is referred to as the scalar potential.

\section{EQUATIONS OF MOTIONS}

Let us first discuss the equations of motions of the fields which can be obtained by varying the action related to the Lagrangian (7) with respect to $g_{\mu \nu}, A_{\mu}^{\Lambda}$, and $z^{i}$. Then, we have three equations. First, the Einstein field equation

$$
R_{\mu v}-{ }^{1}, \ldots=
$$

where the energy-momentum tensor $T_{\mu \nu}$ is given by

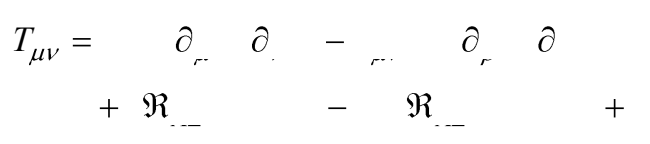

Second, the gauge field equation of motions is

$$
\varepsilon^{\mu \nu \rho \sigma_{\square}} \square_{v}\left(\square_{\Lambda \Sigma} F_{\rho \sigma}^{\Lambda}-\square_{\Lambda \Sigma} \tilde{F}_{\rho \sigma}^{\Sigma}\right)=0,
$$

together with the Bianchi identities

$$
\varepsilon \quad \partial \quad=.
$$

Third, the scalar field equation of motions

$$
\begin{aligned}
& \frac{g_{i j}}{\sqrt{-g}} \square_{v}\left(\sqrt{-g} g^{\mu v} \square_{\mu} z^{j}\right)+\Gamma_{i j k} \square_{\mu} z^{j} \square^{\mu} z^{k} \\
& =\square_{i} \square \Lambda \Sigma F_{\rho \sigma}^{\Lambda} F^{\Sigma \rho \sigma}+\square_{i} \square_{\Lambda \Sigma} F_{\rho \sigma}^{\Lambda} \tilde{F}^{\Sigma \rho \sigma}-\square_{i} V
\end{aligned}
$$

Taking the trace of (12) we finally get

$$
R=\ldots \partial_{\mu} \partial \quad z, \bar{z}
$$

which shows that the Ricci scalar of the spacetimes depends only on the dynamics of the real scalars $z^{i}$. The Ricci scalar (11) becomes a constant if the scalars $z^{i}$ are frozen or in other words $\partial_{r}=$ in particular regions $\mathbf{N} \subseteq \mathbf{M}$ where $\mathbf{M}^{1,3}$ is a solution of (6)-(10) describing a four dimensional curved spacetime. This will be discussed in detail in the following sections. 


\section{DYONIC DOMAIN WALLS}

In this section we particularly discuss a class of domain walls that has both electric and magnetic charges, called dyonic domain wall, in Einstein-Maxwell-Higgs theory. As discussed in the preceding section this case is related to the case in Theorem 2.

To obtain a domain wall solution one has to solve the set of equations of motions (6), (8), and (10) on the metric (1). So, we have three coupled nonlinear differential equations. It is hard to us to find an exact solution in general, but one can only consider the asymptotic solution, namely around $u \rightarrow \pm \infty$. In this limit the scalars $z^{i}$ becomes fixed which are the solution of (6), (8), and (10). In the context of (10) these frozen scalars $z_{0}^{i}$ extremize both the scalar potential $V z$ and the gauge couplings $\Re_{\ldots}$ and $\mathfrak{I} . .$. . Therefore, $z^{i}{ }_{0}$ can be viewed as critical points of the scalar potential $V z$ and the gauge couplings $\mathfrak{R}_{\ldots}$ and $\mathfrak{I} \ldots$. Plugging this $z_{0}^{i}$ into (6) and (11) one concludes that we have spaces of constant scalar curvature which are not Einstein in the asymptotic region. At the level of Ricci scalar (11) the gauge fields $A_{\mu}^{\Lambda}$ does not play an important role since it does not depend $A_{\mu}^{\Lambda}$. Thus, we have a dyonic curve domain walls that interpolate two spaces of different constant scalar curvatures.

\section{CONCLUSIONS}

So far we have considered some aspects of four dimensional curved charged domain walls which interpolates two ground states which are spacetimes of constant Ricci scalar curvatures. These scalar curvatures have different values. In four dimensional EinsteinMaxwell-Higgs theory, these vacua (ground states) lie in the asymptotic regions in which the complex scalars are frozen. In other words, Ricci scalar curvatures are determined by these frozen scalars that belong to moduli spaces. In the future we will extend the analysis to a case of higher dimensional theories including ten dimensional string theories and the mysterious eleven dimensional $\mathrm{M}$ theory.

\section{ACKNOWLEDGMENTS}

This work was supported by Riset KK ITB 2011 No. 222/I.1.C01/PL/2011 and is extended by Riset KK ITB 2013 No. 122.6/AL-J/DIPA/PN/SPK/2013.

\section{REFERENCES}

1 For a review see, for example:

Aharony, O., Gubser, S.S., Maldacena, J., Ooguri, H. and Oz, Y. 2000. Large N Field Theories, String Theory and Gravity. Physics Reports Vol. 323, pp.183.

2 Gunara, B.E., Zen, F.P. and Arianto. 2007. BPS Domain Walls and Vacuum Structure of $\mathrm{N}=1$ Supergravity Coupled to a Chiral Multiplet. Journal of Mathematical Physics Vol. 48, 053505.

3 Gunara, B.E. and Zen, F. P. 2009. Kähler-Ricci Flow, Morse Theory, and Vacuum Structure Deformation of $\mathrm{N}=1$ Supersymmetry in Four Dimensions. Advances in Theoretical and Mathematical Physics Vol. 13, pp. 217-257.

4 Gunara, B.E. and Zen, F. P. 2009. Deformation of Curved BPS Domain Walls and Supersymmetric Flows on 2d Kähler-Ricci Soliton. Communications in Mathematical Physics Vol. 287, pp. 849-866. 
5 Gunara B.E. and Zen F. P. 2009. Flat BPS Domain Walls on 2d Kähler-Ricci Soliton. Journal of Mathematical Physics Vol. 50, 063514.

6 Gunara B.E., Zen F. P. and Arianto. 2011. Spherical Symmetric Dyonic Black Holes and Vacuum Geometries in $4 \mathrm{~d} \mathrm{~N}=1$ Supergravity on Kähler-Ricci Soliton, Reports on Mathematical Physics Vol. 67, pp. 395-413.

7 D'Auria, R. and Ferrara, S. 2001. On Fermion Masses, Gradient Flows and Potential in Supersymmetric Theories. Journal of High Energy Physics, Vol. 0105, pp.034. 